\title{
Anomaly crust fields from MAGSAT satellite measurements: their processing and interpretation
}

\author{
Nina M. Rotanova, Andrei L. Kharitonov and Alfia Kh. Frunze \\ Institute of Terrestrial Magnetism, Ionosphere and Radiowave Propagation (IZMIRAN), \\ Russian Academy of Science, Troitsk (Moscow Region), Russia
}

\begin{abstract}
The space distribution of the magnetic anomaly field for the Pacific Ocean is obtained from data of the satellite MAGSAT. A number of long-wavelength magnetic anomalies of the region are identified. A spectrum analysis of a number of profiles of the anomaly field is performed disclosing typical scales of such anomalies. The wave transform of the anomaly magnetic profiles reveals and explicitly exposes the structure of the considered profile. A schematic complex cross-section is constructed, which demonstrates that the satellite data may be used in the study of the magnetic anomaly.
\end{abstract}

Key words MAGSAT satellite data-magnetic and gravity anomalies - wavelet and maximum entropy analysis - Pacific Ocean - earthquake hypocenters

\section{Introduction}

The MAGSAT satellite data are still extensively used in the study of magnetic anomaly fields. The Danish satellite OERSTED was launched in 1999. However the average altitude of the MAGSAT satellite is approximately $400 \mathrm{~km}$, and the altitude of the new satellite is about $700 \mathrm{~km}$, which makes it considerably more difficult to identify anomaly fields. It seems likely that anomaly fields at such altitudes can be identified only in those cases when they are very intense, as, for example, in the case of the Kursk magnetic anomaly. Therefore, the MAGSAT satellite measurements

Mailing address: Dr. Andrei L. Kharitonov, Institute of Terrestrial Magnetism, Ionosphere and Radiowave Propagation (IZMIRAN), Russian Academy of Science, 142092 Troitsk (Moscow Region), Russia; e-mail: ahariton@izmiran.rssi.ru still remain in force, as confirmed by materials of the last IAGA-IASPEI meeting (Kharitonov and Belikova, 2001). The published maps of anomaly fields obtained by a number of investigators (Arkani-Hamed and Strangway, 1986; ArkaniHamed et al., 1994; Ravat et al., 1995) are presented in color or black and white, they are significantly smoothed out, local anomalies are practically missing on the maps. Such maps cannot be used in performing an independent geophysical interpretation. The goal of the present work is the identification of long-wavelength magnetic anomalies for the Pacific Ocean region, the analysis of their space structure, their correlation with anomaly fields for continents, as well as with other geophysical parameters.

\section{Choice of experimental data, a brief description of the technique for their processing}

As mentioned above, the data from the satellite MAGSAT were used in constructing a space structure of the magnetic anomaly field for the $\mathrm{Pa}$ - 
cific Ocean region. For the territory under consideration with coordinates within latitudes from $55^{\circ} \mathrm{S}$ to $55^{\circ} \mathrm{N}$ and within longitudes from $120^{\circ}$ to $290^{\circ}$, the 1007 morning and 970 evening passes were chosen, for which the geomagnetic activity index $K_{p}<2$. The passes were chosen for the scalar field $B$ and for three components $X, Y, Z$. So, 18700 measurement points of the magnetic field were used for each component of the field within the limits of the territory under consideration.
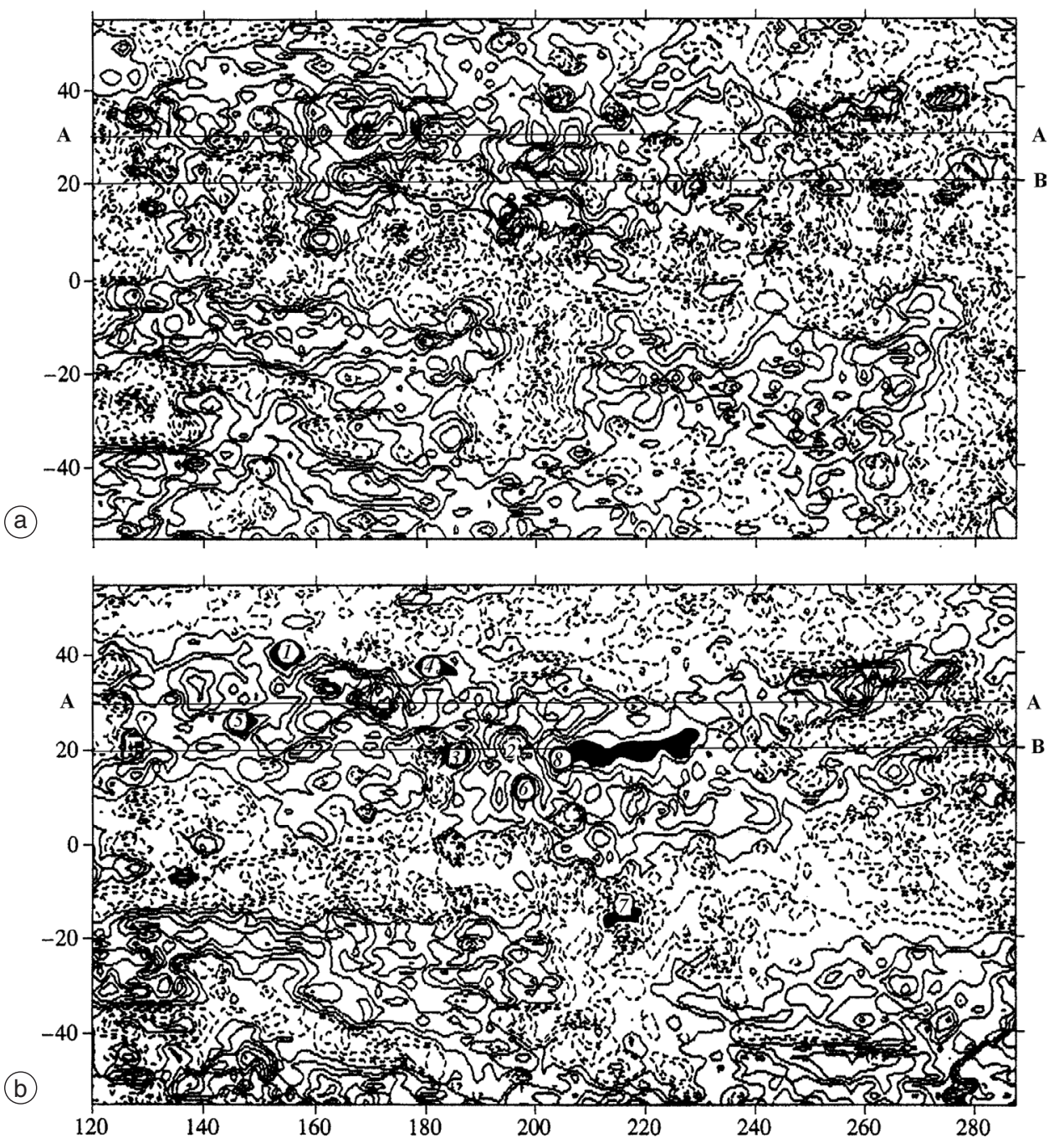

Fig. 1a,b. Spatial structure (a) of the scalar magnetic anomaly field $\Delta B_{a}$ and (b) of the vertical component $\Delta Z_{a}$ obtained from the MAGSAT satellite data for the Pacific Ocean. a) Solid lines denote positive values of the anomaly magnetic field, dotted lines show negative values. Contour interval of $2 \mathrm{nT}$. b) Results of the spectral analysis of the magnetic field profile along satellite pass over the Pacific Ocean area using maximum entropy method. Magnetic anomaly for tectonic uplifts: 1 - Shatsky; 2 - Hawaiian; 3 - Mid-Pacific Ocean; 4 - Hess; 5 Marcus; 6 - Line-15; 7 - Tuamotu; 8 - Molokai Fault. 
We recall briefly that the magnetic field measured at the altitude of the satellite is a complex function of space and time and is caused by various physical sources, among which are the following: processes within fluid parts of the core, responsible for more than $90 \%$ of the measured field, that has been termed the main magnetic field; current systems near the Earth generating the magnetospheric and ionospheric fields; induction fields; and, finally, anomaly fields related to magnetization of the Earth's crust. A rather complex problem arises of extracting the fields measured at the satellite.

With the aid of analytical models for each of the mentioned components the main and magnetospheric-ionospheric fields were calculated, which were removed from the measured values (Rotanova et al., 1997). The residual fields obtained in this way were used in construction of the maps of the anomaly field. For this purpose, the entire territory was divided into $2^{\circ} \times 2^{\circ}$ blocks, and the mean values were calculated within each block, which were taken as the values of the anomaly field. One can find a comprehensive description of this technique for obtaining anomaly fields in Langel and Eastes (1985), Cohen and Achache (1990), Rotanova et al. (1997).

We have constructed numerical maps of the anomaly magnetic field for the Pacific Ocean region from evening and morning passes separately, as well as from the total information for the scalar field and components. Additionally, the map was constructed of differences of the scalar value of the anomaly field from the morning and evening passes reflecting an error, which amounted, on the average, to $2 \mathrm{nT}$.

The space structure of the magnetic anomaly field within the Pacific Ocean for the scalar field $\Delta B_{a}$ and for the vertical component $\Delta Z_{a}$ is shown in fig. 1a,b.

\section{Spatial structure of the magnetic anomaly field of the Pacific Ocean region}

An analysis of the maps presented in fig. $1 \mathrm{a}, \mathrm{b}$ demonstrates that the crustal anomaly field structure in this region is rather complex. A series of positive and negative anomalies of the region is revealed. The general structure of the anomaly field for the region under consideration exhibits a sublatitudinal strike, which manifests itself especially clearly on the map of $\Delta B_{a}$ in the northern part of the Pacific Ocean. The latitudinal strike of anomalies is disturbed near Australia, where a spiral distribution of zones of magnetic anomalies is observed near its central part.

The spatial structure of the anomaly field for the Pacific Ocean differs significantly from similar fields for continents, for example, for North America and Eurasia. High-amplitude anomalies (more than $15 \mathrm{nT}$ at the altitude of the satellite) are revealed almost for the entire territory of the North America. At the same time, from the data of morning and evening passes, the maximum values for the territory under consideration vary within the following limits: $\Delta B_{a}$ from -8 to $+6, \Delta X_{a}$ from -10 to $+11, \Delta Y_{a}$ from -10 to +12 and $\Delta Z_{a}$ from -9 to $+10 \mathrm{nT}$. Numerical values of the field vary somewhat depending on the processing technique used. For the version of the map under consideration, the values of the field are in most cases within the limits from -10 to $+10 \mathrm{nT}$, even though larger values are not excluded.

An analysis of the spatial structure of anomaly fields for the Pacific Ocean region presented in fig. 1a,b permits us to formulate a question concerning the reliability of the identified longwave length anomalies. For this purpose, the constructed maps were compared with the similar results presented in Langel and Eastes (1985), Arkani-Hamed and Strangway (1986), Cohen and Achache (1990), Arkani-Hamed et al. (1994), Ravat et al. (1995) from the MAGSAT satellite data, as well as with the maps obtained from the data of the POGO satellite measurements (Regan et al., 1975). Such a comparison showed that the main large-scale anomalies are well identified from the data of both satellites. Nevertheless, the maps (including those constructed for the Pacific Ocean) constructed from the data of different satellites differ in relation to the different altitudes of the orbits of the satellites. MAGSAT, without question, provides the most detailed structure of the anomaly field, and the anomalies themselves have the highest intensity. Secondly, different 
processing techniques-methods of data filtration are often applied in many works, therefore, the final maps are strongly smoothed off. The Australian and Central Pacific Ocean regions are examples of the most detailed structures of the anomaly field on our maps.

\section{Spectral analysis and wavelet transform of profiles of the anomaly field for the Pacific Ocean area}

In order to interpret magnetic anomaly fields they should be represented not only in the spatial, but also in the frequency domain. Practically all the methods for the solution of forward and inverse problems of the anomaly field are based on the results of spectral analysis. The present work performed numerous calculations of spectra for profiles of the anomaly field iso- lated out of the MAGSAT measurements for the Pacific Ocean area.

All the calculations were carried out by the method of maximum entropy with the use of the following formula:

$$
S(L)=\frac{P_{n}^{2} \Delta X}{\left|1+\sum_{k=1}^{n} r_{n k} \exp (-j 2 \pi \Delta x / L)\right|^{2}}
$$

where $\left(r_{n 1}, \ldots, r_{n n}\right)$ and $P_{n}$ are parameters of the autoregression of the $n$-th order and power of the prediction error; $\Delta X$ is the discretization step of the series analyzed; $L$ represents the period in the spectrum.

Results of numerical calculations for the chosen series of profiles are presented in fig. 2. As one can see from the figure, the main peaks in the spectra of anomaly fields at the satellite

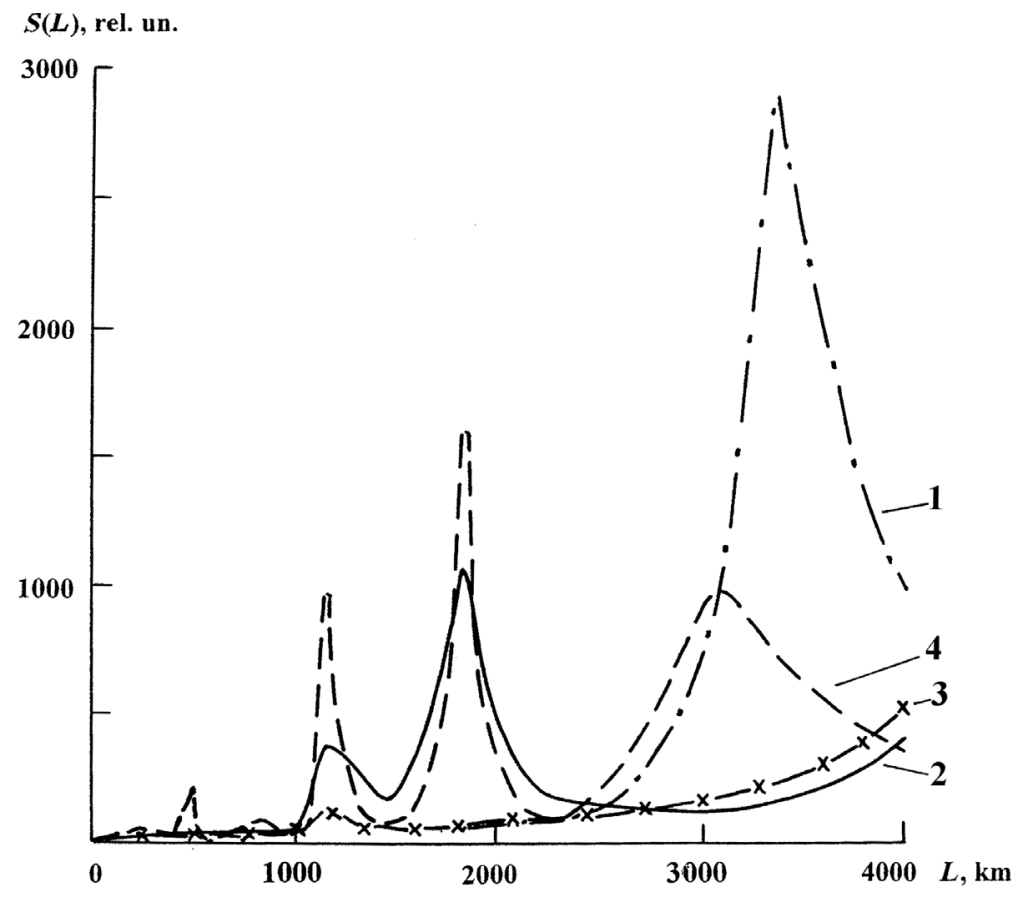

Fig. 2. Results of the spectral analysis of MEM of the magnetic field along satellite passes over the Pacific Ocean area. Periods (in $\mathrm{km}$ ) are plotted as abscissa, spectrum densities (in relative units) - as ordinates. 
altitudes fall within periods approximately from 400 to $4000 \mathrm{~km}$. The periods of large regional anomalies with $L_{1}=400-500 \mathrm{~km}, L_{2}=1000$ $1200 \mathrm{~km}, L_{3}=2000 \mathrm{~km}$ and $L_{4}=3000-3500 \mathrm{~km}$ are the most significant ones. As to the peaks within the periods from 6000 to $8000 \mathrm{~km}$, they require special investigation. As illustrated by Rotanova et al. (1999), the latter are not manifested in all the passes. Therefore, most likely, these peaks are related to residual fields from the main magnetic field and from the fields of the magnetospheric-ionospheric origin. But it should be mentioned that, from the data of hydromagnetic survey in the Pacific Ocean, Nomura (1978) identified anomalies with periods from 600 to $5000 \mathrm{~km}$ as a result of spectrum analysis and related them to the deep regions of the lithosphere.

Unlike the maximum entropy method, the wavelet transform permits us not only to identify characteristic features in a spectrum, but, also, to observe their changes in time or in space. In other words, the wavelet transform provides a two-dimensional distribution of the series under investigation with independent values of its frequency and coordinates. Today, this method finds very extensive application in the analysis of experimental data, because its basis is local and the time-and-frequency window is moveable. In general, the family of continuous wavelets to be analyzed can be described as

$$
\psi_{a, b}(t)=a^{-v} \psi\left(\frac{t-b}{a}\right)
$$

where $a$ is the dilation parameter, $b$ is the translation parameter, and $v$ is the normalizing coefficient equal to $1 / 2$ or 1 . Then the continuous wavelet transform of a signal $f(t)$ has the form

$$
W(a, b)=a^{-v} \int_{-\infty}^{+\infty} \psi^{*}\left(\frac{t-b}{a}\right) f(t) d t
$$

where $\psi(t)$ is a real or complex wavelet, and the asterisk at $\psi(t)$ means the complex conjugation. All the calculations, here, were performed with the aid of the MHAT-analyzing wavelet, an analytical expression of which takes the form

$$
\psi(t)=(1-t)^{2} \cdot \exp \left(-t^{2} / 2\right) .
$$

As initial data we considered longitudinal profiles of the anomaly field over the Pacific Ocean area. One example of such profiles (A-A) along $\varphi=30^{\circ} \mathrm{N}$ is illustrated in fig. 3a. In fig. $3 \mathrm{~b}, \mathrm{c}$ wavelet transforms of this profile are shown representing numerical values of the coefficients $W(a, b)$, where the scale coefficient $a$, which grows linearly, is plotted along the ordinate axis, and the length of the profile in degrees along the horizontal axis. According to Astafieva (1996), the scale coefficient $a$ is related to the characteristic spatial scale by the formula

$$
d=\pi a / \omega_{\psi}
$$

where $\omega_{\psi}=\sqrt{2}$ in the case of the MHAT-analyzing wavelet.

The pattern of the wavelet transform of the basic profile is shown in fig. 3b,c. A series of scale inhomogeneities is revealed along the longitudinal profile exhibiting characteristic dimensions: when the values of the scale coefficient $a$ are small, the dimensions of these peculiarities are $4-5^{\circ}$ with small numerical values of coefficients $W(a, b)$, further longitudinal inhomogeneities with dimensions of $10-20^{\circ}$ are identified, and, finally, large-scale inhomogeneities, the dimensions of which amount to $30-40^{\circ}$. In fact, the wavelet analysis permits us not only to identify a series of classes of long-wavelength anomalies at satellite altitudes, but, also, to determine those longitudes, where these anomalies are observed.

In fig. $3 b$, attention is called to the fact that a scale of about twenty divides the coefficient pattern into two noticeably different regions. Only two large-scale inhomogeneities were identified for the big $a$ in the upper area. The other structure of the field is presented in fig. 3c - the complex structure of the field is observed here with different dimensions of inhomogeneities. Practically all the space dynamics of the magnetic anomaly field is concentrated at a scale inferior to $a \approx 20$.

Comparison of the results of wavelet analysis with the real data presented in fig. 1a,b revealed that the large positive area in $W(a, b)$ 
Profile A - A
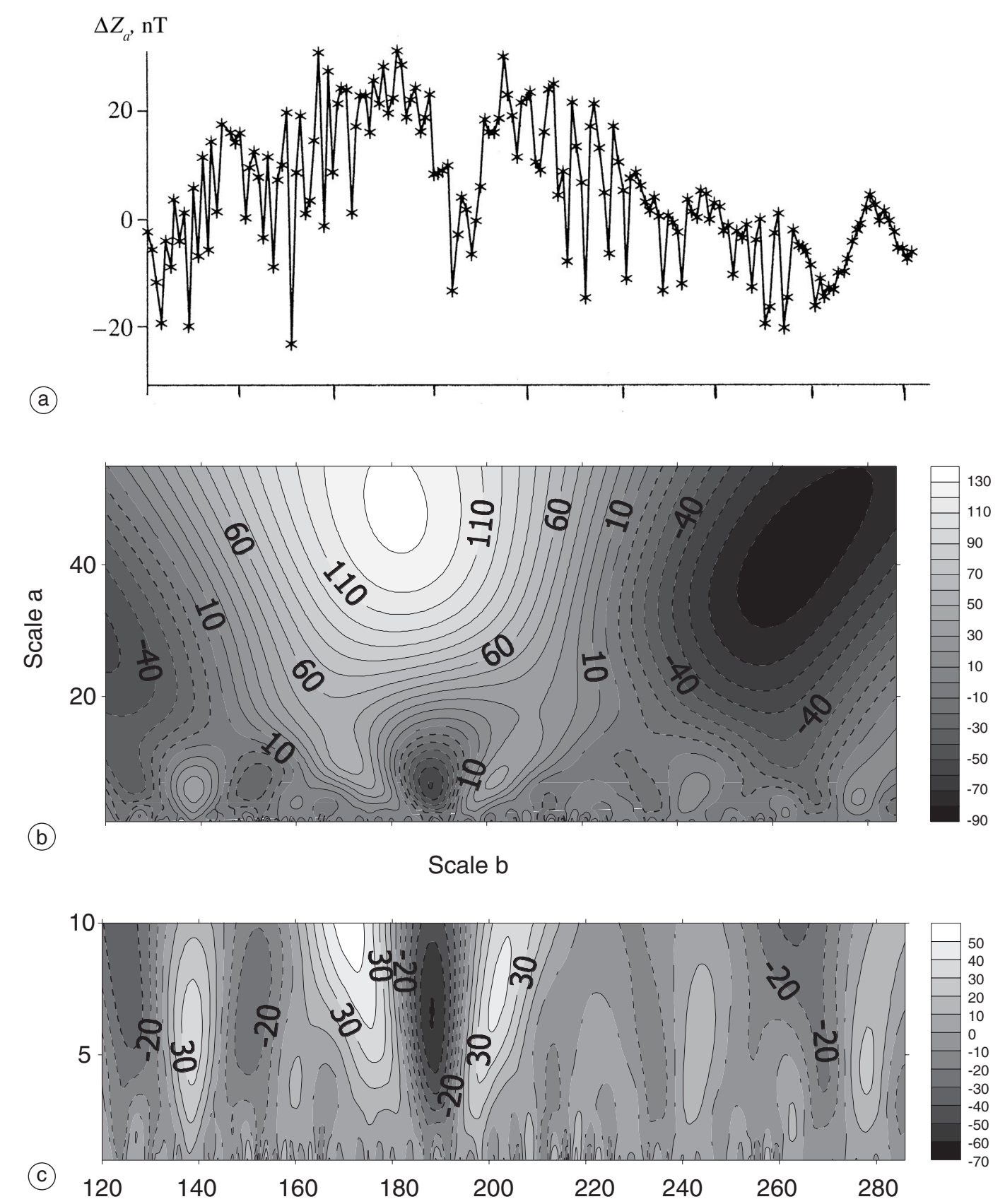

Fig. 3a-c. Profile of the anomaly magnetic field for the Pacific Ocean area along $\varphi=30^{\circ} \mathrm{N}$ (a) and its wavelet transform for different values of the characteristic scale $a(b, c)$. 
corresponds to the large-scale structure of the Pacific Ocean, and the negative one to the large-scale structures of North America. The negative anomaly at the smaller scales of $a$ within the limits of longitudes of $180-200^{\circ}$ corresponds to the Hawaiian uplift.

Thus, the results of one-dimensional spectral analysis and the wavelet transform of the magnetic anomaly profiles from the MAGSAT satellite measurements has demonstrated their complex structure in space; several classes of longwavelength anomalies are identified with different spatial dimensions. The structure of the coefficients of the wavelet transform not only revealed characteristic scales of spatial inhomogeneities in the anomaly field, but also showed their localization at the longitudinal profile. It has been also found that the dynamics of such fields is determined predominantly by smallscale values of the parameter $a$.

\section{Magnetic anomalies of the Pacific Ocean Area from the MAGSAT satellite data and their relationship with the deep-seated structure of the crust and upper mantle}

The relationship between the magnetic anomaly field and tectonic structures within the Earth's crust and the upper mantle is a matter of common knowledge. In recent years, certain progress based on ground (Kolesova, 1985) and satellite observations has been made in this direction. For instance, the data on scalar magnetic fields obtained from the POGO satellite measurements revealed that long-wavelength magnetic anomalies correlate well with largescale tectonic structures (Regan et al., 1975; Frey, 1979; Mayew, 1979; Mayew et al., 1982).

In this regard, anomaly fields obtained at lower orbits of the MAGSAT satellite offer a great advantage. Making use of the global maps of the anomaly field obtained by Langel et al. (1982), Frey (1982) revealed their good agreement with tectonic structures and especially singled out the Shatsky rise located within the westernmost part of the large positive magnetic anomaly of the Hawaiian belt in the Pacific Ocean. The MAGSAT anomaly fields substantially supplement the near-Earth data in determining the structure of the magnetically active layer of the lithosphere. As illustrated by Pashkevich et al. (1994), in many cases, especially when ground measurements are absent, the MAGSAT anomaly fields revealed magnetic inhomogeneities, to establish the contribution of deep-seated sources, to take into account the relationship between ground and satellite anomalies, and, finally, to construct a magnetic model of the lithosphere for a number of regions.

We shall compare the space distribution of anomaly fields with the tectonic structure of the Pacific Ocean area, as well as with other geophysical fields. The total region of the Pacific Ocean is divided into oceanic basins, submarine ridges, deep-sea grooves and continental margins. The schematic representation of this region, obtained in Sorokhtin (1979), is presented in fig. 4. As one can see, island arcs and other uplifts of a greater part of the Pacific Ocean are characterized by negative anomalies of the magnetic field. The eastern part of the Pacific Ocean, especially the Eastern Pacific Ocean uplift, is related to positive anomalies. At the same time, the satellite maps of the anomaly field also provide a clear confirmation for other tectonic structures. The Shatsky rise (1) in the northwestern part of the Pacific Ocean, the Hawaiian uplift (2) in the central part of the Pacific Ocean, the Mid-Pacific Ocean uplift (3) and so on are examples.

The satellite anomaly gravity fields are of significant value for investigation of the structure of the Earth's crust of the ocean. A comprehensive study of such fields was performed by Gainanov and Panteleev (1991). The data on magnetic anomalies extracted from the MAG- SAT satellite measurements, as well as the anomalies of gravity, revealed practically the same tectonic structures over of the Pacific Ocean.

The most comprehensive information on the structure of the lithosphere is given using various geophysical observations. The results of such interpretation of anomalies of the magnetic field together with other geophysical fields along the profile (B-B) at latitude $\varphi=$ $=20^{\circ}$ are presented in fig. 5. Here, curves 1 and 2 correspond to the vertical $\left(\Delta Z_{a}\right)$ component 


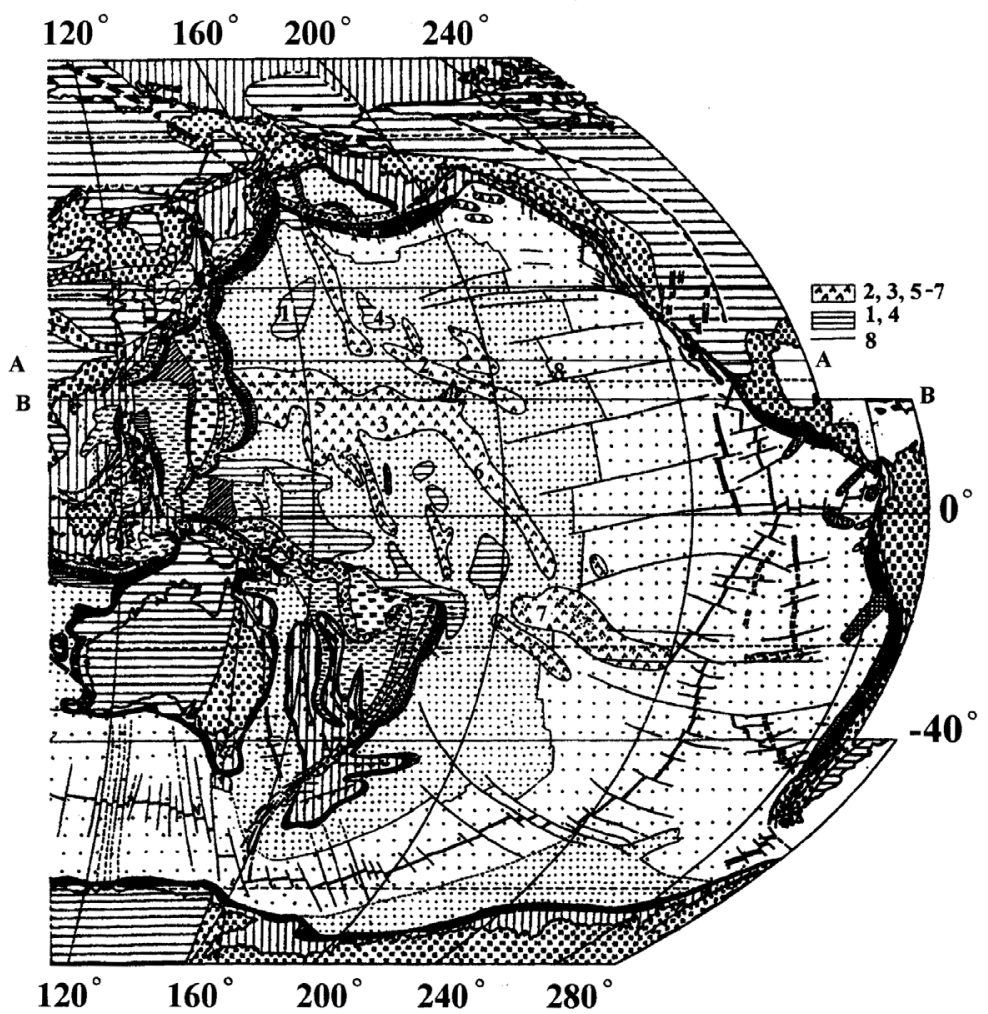

Fig. 4. Tectonic structure of the Pacific Ocean area presented in Sorokhtin (1979). Shaded tectonic areas are zones of uplifts with distribution of volcanic mountains. Uplifts: 1 - Shatsky; 2 - Hawaiian; 3 - Mid-Pacific Ocean; 4 - Hess; 5 - Marcus; 6 - Line-15; 7 - Tuamotu; 8 - Molokai Fault.

and scalar $\left(\Delta B_{a}\right)$ values of the anomaly magnetic field. Curve 3 represents the variation of the gravitational field $\Delta g$ in the Glenni reduction, and curve 4 represents variations of the heat flow (Gorshkov et al., 1974). Under the geophysical fields mentioned, the complex geophysical cross-section of the lithosphere of the Pacific Ocean is shown, including the depth of the sea bottom surface for this profile (curve 5), results of the interpretation of seismic sounding (curve 6), depths of the lower border of the lithosphere from the data on the gravity field (curve 7) (Gainanov et al., 1998), and estimations of the lower edge of the magnetically active layer from the data on the anomaly field from satellite measurements based on the technique developed by Serkerov
(1991) (curve 8). Here, numbers also indicate the densities of different layers of the lithosphere. Zone 9 corresponds to the area of thinning of the magnetic rock.

An analysis of all the geophysical information presented in fig. 5 testifies the complicated litospheric structure in this region. Apparently confirmation by Zonenshin and Kuzmin (1993) is that side by side with hotspots there are whole hot areas on this territory having an extent of some thousands of kilometers. The central and eastern areas of the Pacific Ocean are examples. The satellite data mostly characterize long-wavelength anomalies; therefore many local features of the structure of the lithosphere cannot be reflected in these anomalies. The extracted long-wavelength anomalies from 
Profile B - B

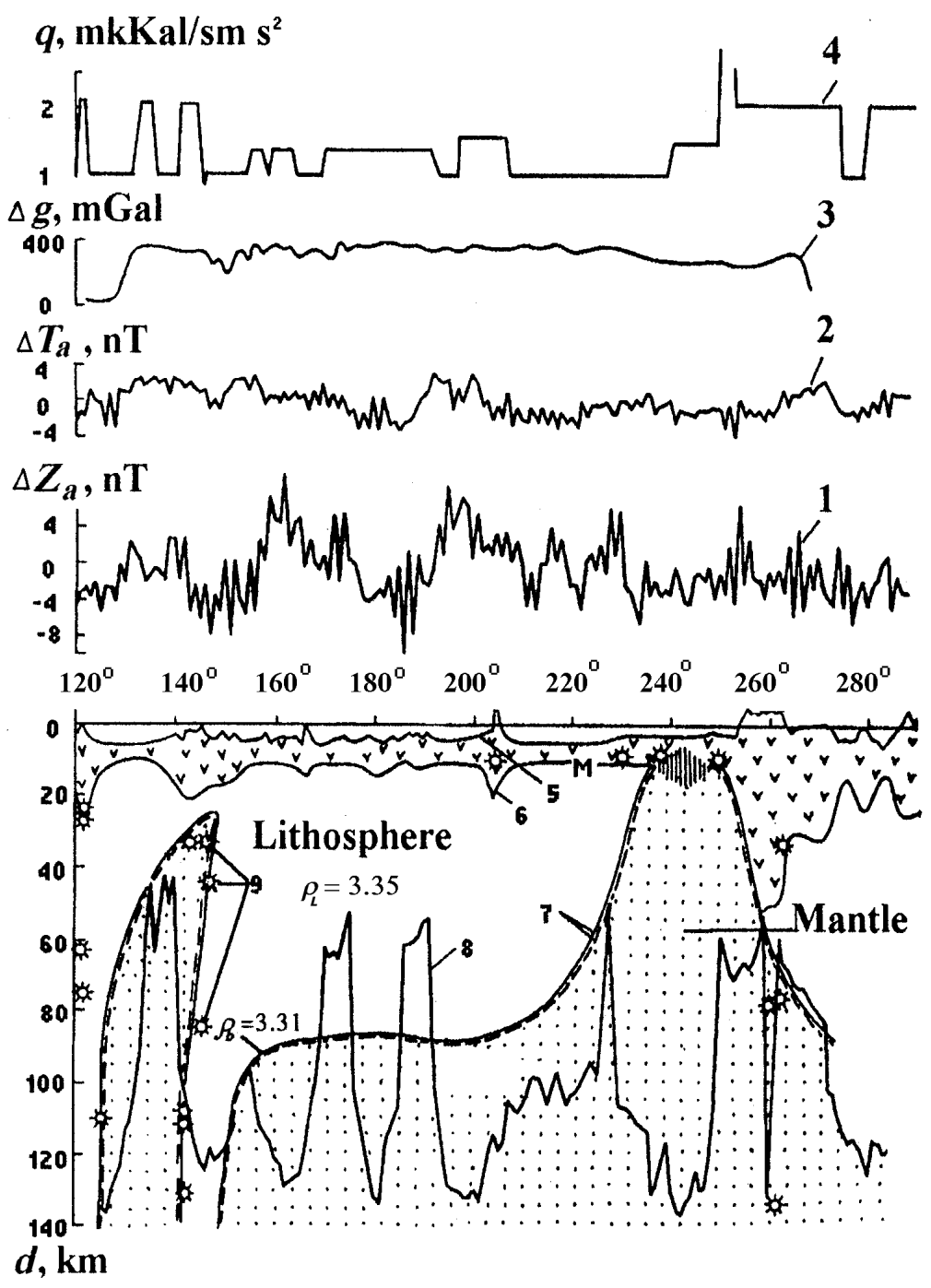

Fig. 5. Complex geophysical cross-section of the lithosphere for the Pacific Ocean region along $\varphi=20^{\circ} \mathrm{N}: 1-$ vertical component of the magnetic anomaly field from the MAGSAT satellite data; 2 - scalar of the magnetic anomaly field from the MAGSAT satellite data; 3 - values of the gravity field in Glenni reduction; 4 - heat flow at the ocean bottom; 5 - depth of the ocean bottom surface; 6 - depth of the Moho surface from the results of interpretation of seismic sounding; 7 - depth of the lower boundary of the lithosphere from the data of the gravity field; 8 - depth of the lower boundary of the magnetically active layer of the lithosphere from the data of satellite magnetic measurements based on the technique proposed by Serkerov (1991); 9 - location of the earthquake hypocenters for the period of the MAGSAT satellite measurements from electronic catalogue of the seismological data of International Geophysical Center of the data of Russian Academy of Sciences. Curves 3, 7 are drawn up from the data of Gainanov et al. (1998): $\rho_{L}$ - density of lithosphere; $\rho_{b}$ - density on the lower boundary of the lithosphere from the data of Gainanov et al. (1998); curves 4, 5, 6 are drawn from the data of Gorshkov et al. (1974). 
the MAGSAT measurements together with gravity observations and with seismic data suggest that their nature in a series of regions of the Pacific Ocean is related to density inhomogeneities within the upper mantle.

Further during the mission lifetime of the MAGSAT satellite from November 1979 through May 1980 the information on all earthquakes taking place within the Pacific Ocean was prepared. The distribution of the depths for such hypocenters of earthquakes was constructed (fig. 6). It is shown that the depths of hypocenters are distributed uniformly in space and by depth. In particular, earthquakes deeper than $50 \mathrm{~km}$ are absent in all the eastern part of the Pacific Ocean adjacent to the American continent, from $\sim 185^{\circ}$ to $250^{\circ}$. Beginning with $\sim 260^{\circ}$ where oceanic plates of the Pacific Ocean (Cocos and Naska) collided with the continental plates of Northern and Southern America there are zones of deeper earthquakes once again. The western part of the Pacific Ocean differs sharply from the eastern part by presence of the intermediate and deep earthquakes, which are located within of subduction zone of the Pacific Ocean plate under continental Australian and Asian plates.

From total information about the earthquakes during the mission of the MAGSAT satellite, we extracted the earthquakes related to a complex cross-section (stars in fig. 5). Figure 5 shows that calculated values of depth of the lower boundary of the magnetically active layer (curve 8) from the anomaly magnetic field in separate zones apparently correlate with the spatial and deep distribution of earthquake hypocenters. In particular, the sharp boundaries between ledges and falls on curve 8 over $\sim 140^{\circ}$ and $\sim 260^{\circ}$ are connected with the earthquake hypocenters. Such boundaries of the lithospheric ledges are observed and in transformed gravitational fields (curve 7). There are sharp changes in the boundary in a magnetic crosssection on the longitudes $\sim 170^{\circ}$ and $185^{\circ}$ existence of which are confirmed by the seismological data (fig. 6).

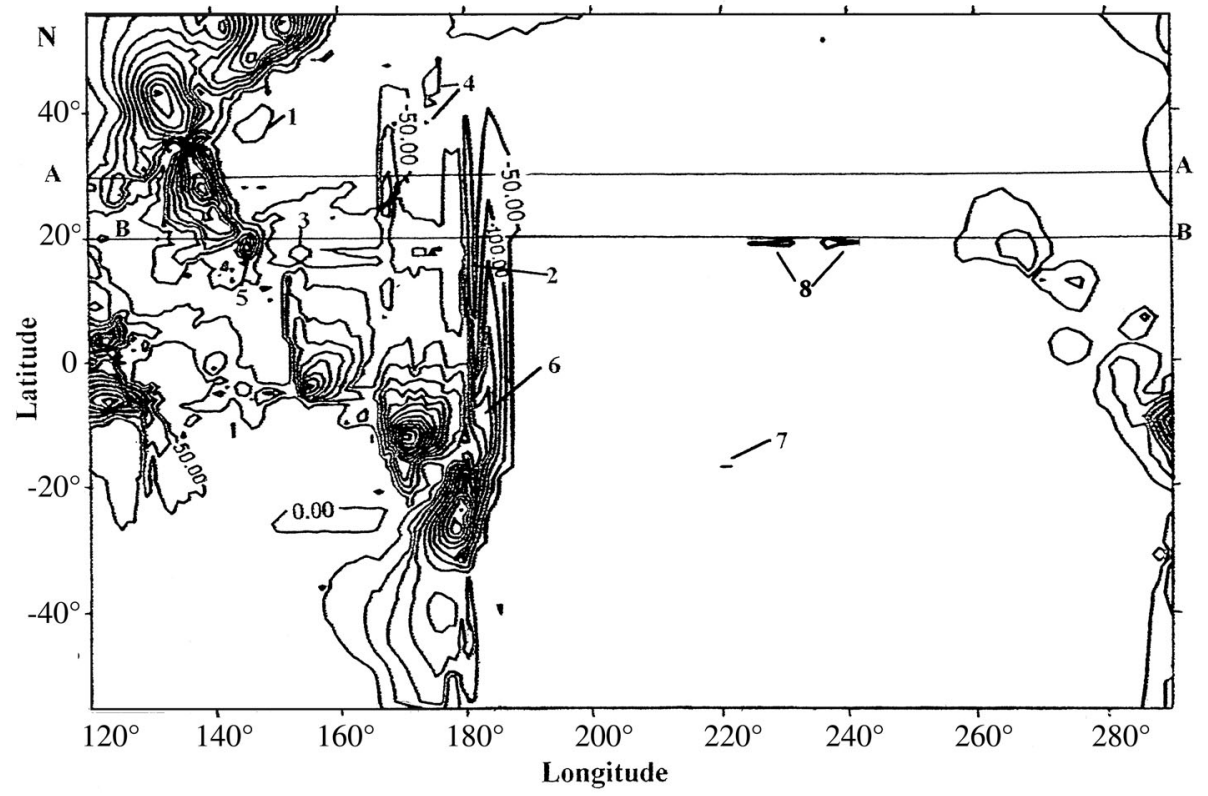

Fig. 6. The map of depth of earthquake hypocenters (in $\mathrm{km}$ ) for the Pacific Ocean region. Uplifts: numbers 18 denote same as fig. $1 \mathrm{a}, \mathrm{b}$. 
It is possible that the boundary edges of the lithospheric layer from the different geophysical data sets over the Pacific Ocean region are connected with the mantle plums penetrating to the Moho boundary (Khain, 1994).

It is necessary to add some facts obtained by geophysicists for this region. Using the magnetic variations from the MAGSAT data the geoelectrical section for the Pacific Ocean sector was constructed, which sharply differs from continental European section. The characteristic peculiarity of the geoelectrical model of the considered region is the presence of thin oceanic layer, the integral conductivity of which is $\sim 2 \cdot 10^{4} \mathrm{~S} / \mathrm{m}$, and also sharp increase of electrical conductivity at the depth $\sim 650$ $\mathrm{km}$. The quantitative estimation of a deep conductive layer conductivity is $\sim 3 \mathrm{~S} / \mathrm{m}$ and its thickness is $\sim 200 \mathrm{~km}$ (Rotanova et al., 1994).

The spatial structure of the geomagnetic secular variations in this region indicates the special role of the Pacific Ocean. We (Rotanova et al.,1982) analyzed the secular variations using the data of the magnetic observatories. It is revealed that for the observatories relating to the Pacific Ocean region amplitudes of such variation have the minimal values compared with similar amplitudes in other regions. Runcorn (1992) tried to explain this fact by the existence of the high-conducting layer in the mantle, which shields on a surface of the Earth secular variations.

At study of conductivity of the lower mantle, lateral heterogeneities were calculated within the range of a spherical layer by the thickness of $700 \mathrm{~km}$ near to the boundary of the core-mantle (Kalugin et al., 1986). A number of anomaly regions was revealed including the large geoelectrical heterogeneity in the region of the Pacific Ocean. It is possible that heterogeneities of conductivity are connected to a spatial structure of the main geomagnetic field and its secular variations. An interaction of a toroidal field with heterogeneities of conductivity leading to intensification of the poloidal field observed on a surface of the Earth can be the possible action of such connection.

\section{Conclusions}

1) From the data of scalar and vector measurements of the magnetic field performed at the MAGSAT satellite, the anomaly maps were constructed, which serve as the basis for investigation of the structure of the magnetically active layer of the region considered.

2) Spectral analysis of the anomaly field was performed for meridian passes over the $\mathrm{Pa}$ cific Ocean water area. Characteristic scales are identified of large long-wave length anomalies with the following values at satellite altitudes (in km): $L_{1} \cong 400-500, L_{2} \cong 1000-1200, L_{3} \cong 2000$ and $L_{4} \cong 3000-3500$.

3) Wavelet transform of the profiles of the anomaly magnetic field for latitude $\varphi=30^{\circ}$ was performed, which permitted us not only to find characteristic spatial inhomogeneities in the transformed fields, but also to show their localization on the profile. Besides, it has been established that the dynamics of the anomaly field manifests mainly on the middle- and smallscale values of parameter $a$.

4) The wavelength magnetic anomalies from the results of the MAGSAT satellite measurements over the Pacific Ocean have been extracted. It is shown that the individual anomalies are reflected in its tectonic structure. A schematic complex cross-section for this region using not only the anomaly magnetic field, but also of other geophysical fields was constructed. Apparently only complex of geophysical parameters and their detailed analysis allow us to understand and investigate the structure of the magnetically active layer of this region.

\section{Acknowledgements}

The authors would like to express their sincere appreciation to Prof. V. Spichak and anonymous reviewers for useful suggestions and comments in preparing the final version of this manuscript.

The authors express their gratitude to the staff members of the laboratory N.I. Volkova and L.I. Yakovleva for their help in preparing the manuscript.

This work was supported by the RFFI grants No. 04-05-64890a and No. 03-05-64656. 


\section{REFERENCES}

Arkani-Hamed, J. and D.W. Strangway (1986): Bandlimited global scalar magnetic anomaly map of the Earth derived from MAGSAT data, J. Geophys. Res., 91, 8193-8203.

Arkani-Hamed, J., R.A. LAngel and M. Purucker (1994): Scalar magnetic anomaly maps of Earth derived from POGO and MAGSAT data, J. Geophys. Res., 99, 2407524090.

AstaFIEVA, N.M. (1996): Wavelet analysis: fundamentals of theory and examples of application, Usp. Phys., 166 (11), 1145-1170.

CoHEN, Y. and J. ACHACHE (1990): New global vector magnetic anomaly maps derived from MAGSAT data, $J$. Geophys. Res., 95, 10783-10800.

FREY, H. (1979): Global geophysics and geology: correlations of satellite-derived and magnetic anomalies with rifts and sutures, EOS, Trans. Am. Geophys. Un., 60, 398-408.

FREY, H. (1982): MAGSAT scalar anomaly distribution: the global perspective, Geophys. Res. Lett., 9 (4), 277-280.

Gainanov, A.G. and V.L. Panteleev (1991): Sea Gravity Prospecting (Nedra, Moscow), p. 215.

Gainanov, A.G., A.V. Bulychev, D.A. Gilod and E.L. MAZO (1995): Gravity field and structure of the tectonosphere of the oceans, Bull. Moscow Soc. Invest. Nature, Ser. Geol., 70 (3), 7-19.

Gorshrov, S.G., V.N. Alekseev, A.I. Rassokho, V.I. Faleev, L.A. Demin and G.M. Khaetov (1974): Atlas of Oceans. The Pacific Ocean (Moscow, Publishing firm of VMF USSR), p. 32.

Kalugin, V.I. N.M. Rotanova and V.P Golovkov (1986): About horizontal heterogeneities of electrical conductivity lower mantle of the Earth, Geomagn. Aeron., 26 (4), pp. 655-659.

Khain, V.E. (1994): Main Problems of Modern Geology (Nauka, Moscow), p. 190.

Kharitonov, A.L. and M.A. Belikova (2001): The Determination of Seismogenic Fault Structures from Satellite Magnetic Measurements (IAGA-IASPEI, Vietnam), p. 440.

Kolesova, V.I. (1985): Analytical Methods of Magnetic Cartography (Nauka, Moscow), p. 222.

LANGEL, R.A. and R.H. EAstes (1985): The near-Earth magnetic field at 1980 determined from MAGSAT, $J$. Geophys. Res., 90, 2495-2510.

LANGel, R.A., J.D. Phillips and R.J. HoRner (1982): Initial scalar anomaly map from MAGSAT, Geophys. Res.
Lett., 9 (4), 269-272.

MAYEW, M.A. (1979): Inversion of satellite magnetic anomaly data, J. Geophys. Res., 45, 119-128.

MaYeW, M.A., H.H. Thomas and P.J. WAsilewsky (1982): Satellite and subsurface expression of anomaly crustal structure in Kentucky and Tenessee, Earth Planet. Sci. Lett., 58 (3), 395-405.

NomurA, M. (1978): Marine geomagnetic anomalies with intermediate wave-length in Western Pacific region, Bull. Ocean. Res. Inst. Univ. Tokyo, 11, 1-42.

PashKeVich, I.K., D.M. PeChersky, A.M. Gorodnitsky, G.M. ValiashKo, V.V. Verba, L.V. VitTe, V.E. VolK, Yu.S. Genshaft, V.M. Gordin, S.V. Eliseeva, S.V. LuKianov, A.V. LiKov, V.S. MARKovsky, M.I. ORLUK, K.V. Popov, E.N. Popov and V.P. SHERbaKov (1994): Petromagnetic Model of the Lithosphere (Naukova Dumka, Kiev), p. 175.

Ravat, D., R.A. Langel, M. Purucker, J. Alkani-Hamed and D.E. AlsDORF (1995): Global vector and scalar MAGSAT magnetic anomaly maps, J. Geophys. Res., 100, 20111-20136.

Regan, R.D., J.C. CAIN and W.M. DAVIS (1975): A global magnetic anomaly map, J. Geophys. Res., 80 (5), 794802

Rotanova, N.M., N.E. Papitashvili and A.N. Pushkov (1982): Spatially-temporary analysis of 60-year's variations of a field under the data of a global network of observatories, Geomagn. Aeron., 12 (5), 847-851.

Rotanova, N.M., V.N.ORAEVSKy, V.Yu. SEMENOV and D. YU. ABRAMOVA (1994): Regional magnetovariational sounding of the Earth using the MAGSAT satellite data, Geomagn. Aeron., 34 (4), 123-129.

Rotanova, N.M., A.L. Kharitonov, A.KH. Frunze and A. ZH. CHANG (1997): The magnetic anomaly field of Asia from MAGSAT satellite data and its physical-geological interpretation, J. Earthquake Predict. Res., 6, 475-494.

Rotanova, N.M., A.L. Kharitonov and A. ZH. Chang (1999): Spectral analysis of the magnetic field measured from the MAGSAT satellite, Geomagn. Aeron., 39 (3), 101-107.

RUNKORN, S.K. (1992): Polar path in geomagnetic reversals, Nature, 356, 654-656.

Serkerov, S.A. (1991): Spectral Analysis in Gravity and Magnetic Prospecting (Nedra, Moscow), p. 280.

SoroKhtin, O.G. (Editor) (1979): Geophysics of the Ocean Geodynamics (Nauka, Moscow), vol. 2, p. 416.

Zonenshain, L.P. and M.I. KuZmin (1993): Deep geodynamics of the Earth, Geol. Geofiz., 34 (4), 3-12. 